\title{
Identification of Sulfavant A as the First Synthetic TREM2 Ligand Discloses a Homeostatic Response of Dendritic Cells After Receptor Engagement
}

\section{Carmela Gallo}

Consiglio Nazionale delle Ricerche

\section{Emiliano Manzo}

Consiglio Nazionale delle Ricerche

\section{Giusi Barra}

Consiglio Nazionale delle Ricerche

\section{Laura Fioretto}

Consiglio Nazionale delle Ricerche

Marcello Ziaco

BIOSERACH SRL

\section{Genoveffa Nuzzo}

Consiglio Nazionale delle Ricerche

\section{Giuliana d'Ippolito}

Consiglio Nazionale delle Ricerche

\section{Francesca Ferrera}

Universita degli Studi di Genova

\section{Paola Contini}

UNIGE: Universita degli Studi di Genova

\section{Daniela Castiglia}

Consiglio Nazionale delle Ricerche

Claudia Angelini

Consiglio Nazionale delle Ricerche

\section{Raffaele De Palma}

Universita degli Studi di Genova

\section{Angelo Fontana ( $\square$ afontana@icb.cnr.it )}

Consiglio Nazionale delle Ricerche https://orcid.org/0000-0002-5453-461X

\section{Research Article}

Keywords: Innate immunity, small molecule, cellular signalling, vaccine adjuvant, neurodegenerative disease, inflammation 
Posted Date: January 11th, 2022

DOI: https://doi.org/10.21203/rs.3.rs-1211312/v1

License: (c) (i) This work is licensed under a Creative Commons Attribution 4.0 International License. Read Full License 


\section{Abstract}

The immune response arises from a fine balance of cellular and molecular mechanisms that provide for surveillance, tolerance, and elimination of dangers as pathogens. Improving the quality of the immune response remains a major goal in immunotherapy and vaccine development. Sulfavant A (SULF A) is a sulfolipid that has shown promising adjuvant activity in a cancer vaccine model. Here we report that SULF A is the first synthetic small molecule binding to the Triggering Receptor Expressed on Myeloid cells-2 (TREM2). The receptor engagement initiates an unconventional maturation of Dendritic cells (DCs) leading to upregulation of the Major Histocompatibility Complex class II (MHC Class II) and costimulatory molecules (CD83, CD86, DC54) without release of T helper type 1 (Th1) or 2 (Th2) cytokines. According to a TREM2 mechanism, this response is mediated by SYK-NFAT axis and is compromised by blockade and gene silencing of the receptor. Activation by SULF A preserved the DC functions to excite the allogeneic T cell response, and induced interleukin-10 (IL-10) release after lipopolysaccharide (LPS) stimulation. These results well support the adjuvant effect of SULF A and offer novel insights into the role of TREM2 in the differentiation of an unprecedented DC phenotype (homeDCs) that contributes to the maintenance of immune homeostasis without compromising lymphocyte activation and immunogenic response. The biological function of SULF-A may be of interest in various physiological and pathological processes involving the immune system.

\section{Introduction}

Pivotal issues in immunology are the ability of the immune system to determine efficient and appropriate responses to pathogens, perform immune surveillance against cancer and clearance of damaged cells. The success of these processes is also dependent on the tolerance to self and the ability to regulate the responses and restore homeostasis once the damage is resolved. Dendritic cells (DCs) are critical effectors of immune system by acting as professional antigen-presenting cells (APCs) [1-3]. Upon activation by engagement of pattern recognition receptors (PRRs), mature DCs stop antigen uptake, start secreting cytokines and increase the expression of surface MHC-peptide complex and co-stimulatory molecules [4]. In this way, DCs integrate signals from the innate and adaptive immune system and shape T cell responses toward either immunity or tolerance $[5,6]$.

In the last years we reported a novel immunomodulatory sulfolipid named Sulfavant A (SULF A) (Figure 1), which primes maturation of DCs derived by human monocytes ( $h$-MoDCs) by a Toll like receptor (TLR)-independent mechanism [7, 8]. In vivo, the sulfolipid elicited antigen specific production of antibodies and proved efficacy as vaccine adjuvant in an experimental model of melanoma [7]. Khameneh et al. have recently underlined the crucial role of Spleen tyrosine kinase (SYK) and Nuclear Factor of Activated T cells (NFAT) in the DC response to non-TLR-based adjuvants, such as MF59, AS03, and Montanide ISA 720 [9]. The intracellular signaling via SYK has become a paradigm in adapative immunity but more recent studies underline its role outside of this context including several functions of innate immune cells and non-immune functions such as osteoclastogenesis [10]. In these 
processes, SYK mediates signaling by specific classes of receptors, such as C-type lectins and triggering receptor expressed on myeloid cells-2 (TREM2) [11], widely present on the DC membrane.

Here we aimed to investigate the effect of SULF A on DC maturation by a reverse pharmacology approach starting from the identification of TREM2 as molecular target of the sulfolipid. Although TREM2 has been implicated in a wide array of immune functions especially related to chronic and degenerative diseases, such as Alzheimer disease and dementia [11-14], various aspects of its signalling in other biological contexts remain poorly understood [11]. Thus, this study also offered the opportunity to investigate role and functional aspects of TREM2 engagement in DCs.

\section{Materials And Methods}

\section{Preparation of T Cells and $h$-MoDCs}

Human monocyte-derived dendritic cells ( $\boldsymbol{h}$-MoDCs) were obtained from Human peripheral blood from leftover blood bags of healthy volunteers collected in different Blood Transfusion Centers of the Campania Region (Italy). No identifying information on the donors was retained. For each assay, human peripheral blood mononuclear cells (PBMCs) were isolated by routine Ficoll density gradient centrifugation (Ficoll Paque Plus, GE Healthcare, USA). Monocytes were purified from PBMCs using MACS CD14 microbeads (Miltenyi Biotech, Auburn, CA, USA) according to the recommendation of the manufacturer. Purity was checked by staining with a FITC-conjugated anti-CD14 antibody (Miltenyi Biotech, Auburn, CA, USA) and FACS analysis and was routinely found to be greater than $98 \%$. CD $14^{+}$ cells were then cultured for 6 days in RPMI medium supplemented with IL-4 $(5 \mathrm{ng} / \mathrm{mL})$ and GM-CSF (100 $\mathrm{ng} / \mathrm{mL}$ ) to differentiate to immature $h$-MoDCs. After harvesting by centrifugation (10 min, $300 \mathrm{~g}$ ), immature $h$-MoDCs were counted and analysed for CD14 /CD83/CD86 ${ }^{+} / \mathrm{HLA}^{-D R^{+}}$expression by FACS. Purity was routinely $>95 \%$. Immature cells were then plated in 12 wells $\left(1 \cdot 10^{6}\right.$ cell $\left./ \mathrm{mL}\right)$ and stimulated with SULF A $(10 \mu \mathrm{g} / \mathrm{mL})$ suspended in PBS at concentration of $1 \mu \mathrm{g} / \mathrm{mL}$. Stimulation with $\mathrm{Pam}_{2} \mathrm{CSK}_{4} 1$ $\mu \mathrm{g} / \mathrm{mL}$ (Invivogen, San Diego, CA, USA) was used as positive control. Naïve CD $4^{+}$and $\mathrm{CD} 8^{+} \mathrm{T}$ lymphocytes were isolated from PBMCs by Naïve Pan T Cell Isolation kit (Miltenyi Biotech, Auburn, CA, USA) according to manufacturer instruction. Purity of naïve T cells was confirmed to be $>95$ $\mathrm{CD}^{2} 5 \mathrm{RA}^{+} / \mathrm{CD} 45 \mathrm{RO}^{\circ} / \mathrm{CCR}^{+}$by flow cytometry.

\section{TREM2 reporter assay}

2B4 GFP-NFAT reporter T cells stably transfected with human TREM2 cDNAs were a gift of prof. Marco Colonna from Washington University in St. Louis. For the binding assay cells ( $2 \times 10^{5} \mathrm{cells} /$ well) were plated in flat 96-well plate in $0.2 \mathrm{~mL} /$ well of RPMI medium and incubated overnight. $\mathrm{MeOH}$ was used as solvent to dissolve compounds and by performing the coating of the plate with $0.05 \mathrm{~mL}$ of solution of lipids at indicated concentration. Reporter cells activity was assessed after overnight incubation as percentage of $\mathrm{GFP}^{+}$cells subtracted from background (vehicle controls) measured by flow cytometry. Data were acquired by BD C6 ACCURI instruments (BD Bioscience, Frankin Lake, NJ, USA). 


\section{Dectin 1 reporter assay}

HEK-Blue ${ }^{\text {TM }}$ hDectin-1b Cells (SEAP reporter cells) (Invivogen, San Diego, CA, USA) were plated in 96-well plates at $4 \times 10^{5}$ cells/well in $0.2 \mathrm{~mL} /$ well of HEK-Blue ${ }^{\mathrm{TM}}$ medium (Invivogen, San Diego, CA, USA) and incubated overnight. $\mathrm{MeOH}$ was used as solvent to dissolve compounds and by performing the coating of the plate with $0.05 \mathrm{~mL}$ of solution of lipids at indicated concentration. Reporter cells activity was assessed after overnight incubation as quantification of secreted alkaline phosphatase (SEAP) released in the medium by reading the optical density (OD) at $655 \mathrm{~nm}$. Zymosan was used as positive control under the same conditions at concentration of $100 \mu \mathrm{g} / \mathrm{mL}$ according to manufacturer's instructions. Quantitative data $(\mathrm{ng} / \mathrm{mL})$ were obtained by a standard curve for the SEAP protein.

\section{Flow Cytometry}

$h$-MoDCs were stained to check the maturation state and the purity with conjugated mouse anti-human HLA-DR BV786, CD83 BUV737, CD86 BV650, CD14 FITC, CD11c PE monoclonal antibodies (BD Bioscience, Frankin Lake, NJ, USA) according to standard protocol. Before acquisition, all cells were incubated with Propidium iodide solution (Thermo Fisher Scientific, Waltham, MA, USA) for dead cell labelling for $5 \mathrm{~min}$ at room temperature. Data were acquired by an ARIA or BD C6 ACCURI flow cytometers (BD Bioscience, Frankin Lake, NJ, USA) and analysed with FlowJo 9 software (Tree Star, Inc., Ashland, OR, USA) and BD C6 ACCURI software.

\section{Real Time PCR}

Total RNA was isolated using Trizol Reagent, according to the manufacturer's protocol. RNA quantity and purity were measured with a NanoDrop 2000 spectrophotometer (Thermo Fisher Scientific, Waltham, MA, USA). Sample purity was checked by A260/A280 ratios between 1.80 and 2.00. Extracted RNAs from all preparations were in this range. Cytokines mRNA expression was measured by quantitative Real TimePCR by using the following primers for DAP12: 5'- GACCCGGAAACAGCGTATCA- 3' (sense), and 5'GTAGACATCCGACCTCTGACC- 3' (antisense); NFAT1: 5'- ATCTGCAGCATCCCAGTGAC- 3' (sense) and 5'CGCAGCTCGTAAGAGCCTG-3' (antisense); NFkß-p5: 5'- GATCTGCCGAGTGAACCGAA-3' (sense) and 5'CAATGTCCTCTTTCTGCACCTT- 3' (antisense); SYK: 5'-CTGGCAGCTAGTCGAGCATT-3' (sense) and 5'ACATTTCCCTGTGTGCCGAT-3' (antisense); RELA: 5'-GAGACACACCACTGCACCTA-3'(sense) and 5'GCTCAATGACACTGGTCTGC-3'(antisense); TREM2: 5'-GCACTCTCACCATTACGCTG-3' (sense) and 5'CTGGTAGAGACCCGCATCAT-3' (antisense); IL12p40: 5'-GCCCATTGAGGTCATGGTG-3' (sense) and 5'TCTTGGGTGGGTCAGGTTTG-3' (antisense); IL-4: 5'-CGACTGCACAGCAGTTCCAC-3' (sense) and 5'CACAGGACAGGAATTCAAGCC-3' (antisense); 18S: 5'-GGCGACGACCCATTCGAAC-3' (sense) and 5'AGGCACGGCGACTACCATC-3' (antisense); IL-6: 5'-CCCTGAGAAAGGAGACATGTAAC-3' (sense) and 5'CCCTGAGAAAGGAGACATGTAAC-3' (antisense); IL-10: 5'-GGGAGAACCTGAAGACCCTC-3' (sense) and 5'AAGAAATCGATGACAGCGCC-3' (antisense); IFNy: 5'-ATGGCTGAACTGTCGCCAG-3' (sense) and 5'TGCAGGCAGGACAACCATT-3' (antisense); NFAT2: 5'- GGTTCAGCTCCATGGCTACA-3' (sense) and 5'AAGATCTGAAGTCCCAGAGGC-3' (antisense). 


\section{ELISA}

ELISA assays for measuring IL-2, IL-12p70, IFNG, IL-10, IL-4 and IL-23 in supernatants were performed using commercially available kits (Thermo Fisher Scientific, Waltham, MA, USA) following the manufacturer instructions.

\section{Surface binding blocking antibody analysis}

Goat anti-human TREM2 antibody (AF1828; R\&D Systems, Milan, Italy) was selected as blocking antibody (bAb) according to Quant et al [15]. Immature $h$-MoDCs were obtained as reported below from 3 donors and incubated for the maturation test with $0.1,0.2,0.5$ and $1 \mu \mathrm{g} \mathrm{mL}^{-1}$ of bAb. Cell concentration was adjusted at $1 \times 10^{6}$ cells $/ \mathrm{mL}$ and the assay was performed for $24 \mathrm{~h}$ in 12 well plates. Cells were then stained for flow cytometry analysis as described above and for TREM2 expression by human TREM2 APC-conjugated antibody (R\&D Systems, Milan, Italy). To an aliquot of treated cells $\left(2.5 \times 10^{5}\right.$ cells), biotin-conjugated rabbit anti goat IgG (Dako F0056, Denmark, 1:1000) was added prior to staining by streptavidin for $30 \mathrm{~min}$ at $2-8^{\circ} \mathrm{C}$.

\section{TREM2 neutralization by bAb}

Immature $h-\mathrm{MoDC}$ were prepared from 3 donors. For the assay, $1 \times 10^{6}$ cells in triplicate for each donor were incubated with $1 \mu \mathrm{g} / \mathrm{mL}$ goat anti-human TREM2 antibody as described before. After incubation at $37^{\circ} \mathrm{C}$ for $60 \mathrm{~min}, 10 \mu \mathrm{g} / \mathrm{mL}$ SULF- A was added in triplicate to samples. Cells were kept at $37^{\circ} \mathrm{C}$ for $24 \mathrm{~h}$ and then harvested and stained as described above for analysis by flow cytometry. As negative control, polyclonal normal goat IgG (isotype control) (AB-108-C; R\&D Systems, Milan, Italy) was used at the same concentration of bAb. An aliquot of cells was used for RNA extraction and RT-PCR analysis of IL-12p40 in triplicate as described above.

\section{siRNA Validation}

A small interfering oligonucleotide (5'-GGAAGAUGAUGGGAGGAAAtt-3') that targets the sequence of human TREM2 was purchased by Ambion (Thermo Fisher Scientific, Waltham, MA, USA) together with a non-targeting siRNA (5'-UUUCCUCCCAUCAUCUUCCtt-3') that was used as negative control. Stock solutions of both siRNA at a concentration of $10 \mu \mathrm{M}$ were obtained in nuclease-free water. Immature $h \mathrm{MoDC}\left(1 \times 10^{6}\right.$ cells) were transfected with 50,100 and $200 \mathrm{nM}$ of siRNA by electroporation using the Amaxa Human Dendritic Cell Nucleofector Kit (Lonza, Basel, Switzerland) following the instructions of the manufacturer and the U-02 Amaxa program (Lonza, Basel, Switzerland). The transfected cells were seeded at $1 \times 10^{6}$ cells/well into 12 -well plates and cultured for $24 \mathrm{~h}$. At this time cells were collected for RT-PCR, flow cytometry and vitality by propidium iodide (PI). In a second experiment, immature $h$ MoDCs $\left(1 \times 10^{6}\right.$ cells) were transfected with $200 \mathrm{nM}$ siRNA. The cells were then recovered at 6,12 and $24 \mathrm{~h}$ and analyzed for RT-PCR, flow cytometry and stained with conjugated TREM2-APC antibody (R\&D Systems) following the manufacturer's instructions. 


\section{Interference of TREM2 by siRNA}

Immature $h$-MoDCs $\left(1 \times 10^{6}\right.$ cells) were incubated with either 200 nM TREM2 siRNA or $200 \mathrm{nM}$ nontargeting siRNA. Cells were seeded at $1 \times 10^{6}$ cells/well into 12 -well plates and cultured $12 \mathrm{~h}$ prior to be stimulated by $10 \mu \mathrm{g} / \mathrm{mL}$ SULF A. After other $24 \mathrm{~h}$, treated $h$-MoDCs were recovered and analysed by flow cytometry and gene expression.

\section{Costimulation by SULF A and LPS}

Immature $h$-MoDCs were prepared and checked for purity as described above. Cells were plated in 12 wells $\left(1 \cdot 10^{6} \mathrm{cell} / \mathrm{mL}\right)$ and treated by $10 \mu \mathrm{g} / \mathrm{mL}$ SULF A. After $30 \mathrm{~min}, 5 \mathrm{ng} / \mathrm{mL}$ LPS was added and plates were incubated at $37^{\circ} \mathrm{C}$ for further $48 \mathrm{~h}$. After this time, cells and supernatants were recovered and analyzed by flow cytometry, gene expression and ELISA.

\section{Allogeneic mixed lymphocyte reaction (MLR)}

Allogeneic mixed lymphocyte reaction (MLR) experiments were conducted using naïve $\mathrm{CD} 3^{+} \mathrm{T}$ lymphocytes (responders) and DCs (stimulators) from 7 healthy donors different from whom monocytes were collected. The MLR was performed by seeding $1 \times 10^{4}$ stimulators (DCs) and $1 \times 10^{5}$ responders (T cells) at 1:10 ratio in round bottom 96 well plates in RPMI medium completed with $10 \%$ human $A B$ serum. Before co-culture, naïve T cells were counted and labelled by carboxyfluorescein succinimidyl (CFSE) ester assay (Thermo Fisher). After 2 washes in PBS 5\% FBS, cells were used for MLR experiments. Unstimulated CFSE labelled lymphocytes were used to set the gate for CFSE control. Three replicates of stimulation with $10 \mu \mathrm{g} / \mathrm{mL}$ SULF A or $1 \mu \mathrm{g} / \mathrm{mL}$ phytohemagglutinin (PHA) were set for each donor. T cells proliferation was analysed by dilution of the CFSE signal using flow cytometry (FACS ARIA; BD Bioscience, Frankin Lake, NJ, USA).

\section{Statistical analysis}

For all markers analyzed by flow cytometry, isotype controls were used by setting gates between 0.5 and $1 \%$ positive events. Statistical analysis to compare the mean values for two groups was performed using non-parametric (two-sample) Wilcoxon test or T-test. The paired version of the test was used when replicates were matched in the two conditions. For multiple groups, we used a one-way or two-way ANOVA with repeated measures (RM ANOVA). followed by post-hoc analysis with a series of pairwise Ttests. We used $\mathrm{BH}$ correction or Tukey test for multiple comparisons. We also fitted linear-mixed models (LMM) when technical replicates were considered. A p-value less than 0.05 was considered statistically significant. Graphics were drawn by GraphPad Prism 8 (GraphPad Software, San Diego California, USA).

\section{Results}

SULF A induces selective TREM2 signalling on reporter cell lines 
Since its discovery, the identification of endogenous ligands of TREM2 has proven elusive although there is an emerging pattern of evidence suggesting that the receptor can interact with a wide array of negatively charged molecules mostly associated to tissue damage or cellular stress [16-19]. In order to test the binding of SULF A to TREM2, we used a consolidated TREM2-reporter cell line that transduces receptor engagement by synthesis of GFP [17]. After incubation with SULF A, the receptor activation was measured as $\mathrm{GFP}^{+}$cells by flow cytometry in comparison to a polyclonal anti-TREM2 antibody (Ab) and phosphatidylserine (PS), which is one of the suggested bona fide ligands [20] (Fig. 2A). The response was dose-dependent in the range from $1 \mathrm{ng} / \mathrm{mL}$ to $120 \mu \mathrm{g} / \mathrm{mL}$ of SULF A with an activity that was higher than that of PS and comparable to anti-TREM2 antibody binding (Fig. 2B). The dose-response curve of the cellbased assay showed a sigmoid shape with an $\mathrm{EC}_{50}$ of $35.9 \mu \mathrm{g} / \mathrm{mL}$ (Supplementary Fig. 1). Conversely, in the same range of concentrations, SULF A failed to stimulate reporter cells for CLEC7A (Fig. 2C), a glycoprotein of the C-type lectin family, and R47HTREM2 (Fig. 2D), a variant of TREM2 that is known to weakly bind PS and other lipid ligands in microglia $[13,17,20]$. These results proved that SULF A binds to TREM 2 in a selective manner and ruled out the non-specific response of the reporter cells.

\section{Transcriptional regulation of intracellular TREM2 signaling by SULF A}

The downstream cascade signal of TREM2 is mediated by the electrostatic interaction with DNAXactivation protein 12 (DAP12) $[11,13]$ that, upon phosphorylation, recruits SYK and initiates the intracellular pathway. The next steps are not fully elucidated but independent authors reported that TREM2-mediated activity depends on NFAT [17, 21, 22], a family of five transcription factors which are mostly regulated by $\mathrm{Ca}^{2+}$. In striking contrast to the response to $\mathrm{C}$-type lectins, such as dectins or mincle, that requires NFAT together with canonical and non-canonical pathways of nuclear factor-kB (NF$\mathrm{kB}$ ) in DCs and macrophages [23][24], TREM2 signaling transduction is independent of NF-kB in DCs [25]. Thus, in order to uncover the signalling originated by SULF A and differentiate between the intracellular pathways of TREM2 and Dectins/Mincle, we carried out a transcriptional analysis of the target genes DAP12, SYK, NFAT1, NFAT2 together with the response of the inducible transcription factors RELA (also called NF-KB p65) and NF-KB p52 that are related to the canonical and non-canonical NF-kB pathways. After $3 \mathrm{~h}$ from the stimulation by $10 \mu \mathrm{g} / \mathrm{mL}$ SULF A, $h$-MoDC showed significant upregulation (expression fold change above 3 ) of SYK and NFAT1, while transcriptional levels of NFAT2 were affected to a lesser extent (Fig. 2E). SULF A did not vary the expression levels of TREM 2 (data not shown), which agrees with the unchanged transcription of DAP12 that is the related cytoplasmic signal-transducing element. Furthermore, NF-KB pathway was unaffected by the sulfolipid while RELA expression was increased by the addition of the TLR agonists LPS or $\mathrm{PAM}_{2} \mathrm{CSK}_{4}$ (Supplementary Fig. 2). Transcriptional analysis over $24 \mathrm{~h}$ revealed the return to basal or near-basal levels of SYK and NFAT1 expression after $6 \mathrm{~h}$ from the addition of SULF A (Supplementary Fig. 3). These data were consistent with the recruitment of SYK that elicits a transient mobilization of calcium pathway and calcineurin-dependent activation of NFAT [26], thus providing support to the downstream pathway depicted in Fig. 2F. It is well-known that NFAT1 transcription factor regulates specific functions of DCs such as tolerance and T cell differentiation [27, 
28], however to the best of our knowledge this is the first direct evidence in support of this signalling in DC upon TREM2 engagement.

\section{Functional response of $h$-MoDCs to SULF A}

We have previously reported that stimulation of $h$-MoDCs by SULF A induces upregulation of HLA-DR and costimulatory molecule CD83, transcription of IL-12p40 (also named IL-12a) and IFN-y genes after $24 \mathrm{~h}[7$, 8]. $h$-MoDCs are primary cultures derived from human monocytes and widely used as cell model of APC. In line to our previous report, at 24 hours stimulation by $10 \mu \mathrm{g} / \mathrm{mL}$ SULF A induced an upregulation of MHC Class II (HLA-DR) and key costimulatory molecules CD86 and CD83 in $h$-MoDCs from 6 human donors (Fig. 3A), with unequivocal evidence on the modulatory effect of the sulfolipid on the costimulus. Within 24h from the treatment (Fig. 3B), there was the significant increase of the transcription of $I L-12 a$ and, at lower extent, of $I L-4, I L-6$, and $I F N g$ while no signal was detected for $I L-10, I L-23 p 19$ and TGF 1 (not shown). However, ELISA on the supernatants after $48 \mathrm{~h}$ revealed only low and variable secretion of IL6 [29] without production of Th1-type or Th2-type cytokines (Fig. 3C). This maturation phenotype of DCs is unconventional for the divergence between the marked stimulatory effect on surface molecules related to $T$ cell signalling and the hyporesponsiveness of cytokine patterns related to typical inflammatory or tolerogenic signatures.

\section{Neutralization of SULF A activity by antibody blocking of TREM2}

To confirm that TREM2 signalling is responsible for the DC maturation induced by SULF A, we carried out an antibody blocking assay, a widely accepted method to determine the receptors involved in biological response. Cells are preincubated with the blocking antibody $(\mathrm{bAb})$ and then stimulated with the putative ligand, whereby decreased activity against targets is attributed to the blocked receptor. In our case, we used a polyclonal goat anti-human TREM2 IgG (TREM2-bAb) that binds the ectodomain of the protein[15]. Prior to start the experiment with SULF A, the effect of TREM2-bAb was tested on $h$-MoDCs from 3 donors. The antibody did not induce DC maturation between 0.1 and $1 \mu \mathrm{g} / \mathrm{mL}$. In this range of concentrations, levels of HLA-DR, CD83 and CD86 were unaffected in comparison to control cells (Supplementary Fig. 4), whereas a dose dependent reduction of the free receptor on the DC surface was verified by an anti-TREM2 antibody by flow cytometry (Fig. 4A). The occupancy of the receptor on the cell surface of $h$-MoDCs was linear with the increase of the concentration of anti-human TREM2-bAb up to $1 \mathrm{mg} / \mathrm{mL}$. At this concentration, the level of the detectable receptor was as low as the levels observed after treatment of DCs by the TLR-4 agonist LPS, which is known to induce TREM2 suppression in microglia [30]. On the contrary, the immunocytochemical analysis by anti-goat biotin secondary antibody (1:1000) followed by staining with streptavidin confirmed the presence of the TREM2-bAb with an increase of the fluorescence linearly related to the concentration (Supplementary Fig. 5). We next evaluated the inhibitory effects of TREM2-bAb on the expression of MHC II and costimulatory molecules induced by SULF A. As shown in Fig. 4B for HLA-DR ${ }^{+} C D 83^{+}$hMoDC cells, flow cytometry analysis revealed that pre-incubation of $h-\mathrm{MoDCs}$ with the blocking antibody reduced the activity of $10 \mathrm{mg} / \mathrm{mL}$ SULF A. The effect was similar on the co-stimulatory molecules CD83 and CD86 
(Supplementary Fig. 6) and the statistical analysis on different donors revealed a dose dependentresponse with $100 \%$ inhibition at $1 \mu \mathrm{g} / \mathrm{mL}$ TREM2-bAb (Fig. 4C). Since the blocking antibody did not initiate TREM2 signalling, the inhibition of SULF A-induced DC maturation was due to the non-functional occupation of the receptor. In contrast, treatment with an isotype control did not affect the stimulatory activity of the sulfolipid (Supplementary Fig. 7). These results suggested that the mechanism of action of SULF $A$ is mediated by TREM2 in a specific manner.

\section{Inhibition of SULF A activity by gene silencing of TREM2}

As a further demonstration of the role of TREM2, we tested the correlation between TREM2 expression levels and SULF A-dependent maturation of $h$-MoDCs. First, we verified the efficiency of a TREM2 siRNA to knock down mRNA and protein levels of the receptor. As shown in Fig. 4D, TREM2 expression was significantly reduced in interfered $h-\mathrm{MoDCs}$, with a down-regulation that was linearly dependent on the concentration of the interfering oligonucleotide. The effect of transfection was optimal with $200 \mathrm{nM}$ SiRNA at $12 \mathrm{~h}$ when we observed reduction of TREM2 gene together with no significant change of the cell vitality (over $80 \%$ ). At this concentration, expression of the phenotypic markers HLA-DR, CD83 and CD86 was unaffected in comparison to both untreated cells and cells transfected with non-targeting siRNA control, thus ruling out non-specific effects (Supplementary Fig. 8). On the other hand, staining by conjugated TREM2-APC antibody showed a reduction of the receptor occurrence on cell surface of $h$ MoDCs interfered with TREM2-siRNA (Supplementary Fig. 9). Incubation of these cells with $10 \mu \mathrm{g} / \mathrm{mL}$ SULF A revealed a clear reduction of the effect of the sulfolipid on the expression of HLA-DR, CD83 and CD86 (Fig. 4E). In analogy with the effect of the blocking antibody reported above, the depletion of the receptor on the cell surface prevented the phenotypic maturation pattern induced by SULF A, which is fully consistent with a TREM2-dependent response of $h$-MoDCs to the sulfolipid.

\section{Effect of SULF A on TREM2-TLR crosstalk.}

TREM2 signalling has acquired relevance in the context of tissue damage and cross-talk with other pathways activated by danger signals [11]. Thus, in order to provide a functional clue on TREM2 activation by SULF A, we tested the molecule for the ability to modify the DC response to TLR agonist. LPS strongly down-regulated TREM2 expression on the surface of $h$-MoDCs. Considering the low amount of residual TREM2, stimulation by SULF A had no detectable effect on these cells (not shown). On the contrary, a 30 minutes preincubation of $h$-MoDCs with $10 \mu \mathrm{g} / \mathrm{mL}$ SULF A followed by $5 \mathrm{ng} / \mathrm{mL}$ LPS (Fig. 5A) downregulated the expression of HLA-DR and the costimulatory markers CD83 and CD86 in comparison to control stimulated only by LPS (Supplementary Fig. 10). After 24h, combination of SULF A and LPS increased production of proinflammatory cytokines IL-12, INFg, and IL-23 in comparison to LPS alone but also triggered strong synthesis of anti-inflammatory IL-10 (Fig. 5B). On the contrary, sequential addition of SULF A and LPS did not affect the synthesis of IL- 6 by $h$-MoDCs, as well as suboptimal concentration of the sulfolipid $(1 \mu \mathrm{g} / \mathrm{mL})$ did not modify the response to LPS. IL-10 can directly attenuate inflammatory profile of DC and macrophages by an autocrine mechanism [31, 32], as well as reduce the excessive proinflammatory responses as part of a negative-feedback mechanism to limit an 
overactive immune response in tissues during infection [33]. Taken together, these data suggest that SULF A tunes DC response to inflammatory signals by a delayed release of IL-10, which is consistent with a mechanism of homeostasis aiming to ensure an appropriate and balanced immune activation.

\section{T cell priming and differentiation by SULF A-stimulated DCs}

Instruction of T lymphocyte response is the hallmark of $\mathrm{DC}$ function. Due to the $\mathrm{DC}$ ability to modulate activation and function profile of T cells, DC represent a main target to improve manipulation of immune responses and vaccine development $[34,35]$. In consideration of the unconventional DC phenotype induced by SULF A, we tested the sulfolipid effect on priming and function of T lymphocytes in a mixed lymphocyte reaction (MLR). After excluding direct activation of T lymphocytes by SULF A, $h$-MoDCs (stimulators) and naïve T cells (responders) from seven different healthy donors were cocultured to determine T cell proliferation and cytokine release. As shown in Fig. $\mathbf{5 C}$, the addition of SULF A to the MLR increased lymphocyte proliferation in comparison to unstimulated cocultures (control). The effect was comparable to the ex vivo expansion induced by the mitogen phytohemagglutinin (PHA) used as positive control. Measurements of the cytokines in the supernatant of the MLR showed that SULF A upregulated production of IL-2 and IL-4 (Fig. 5D-E). Both molecules are pleiotropic cytokines and key signals in growth, differentiation and in vivo homeostasis of T cells[36, 37], yet their functions can be highly divergent and context dependent. The levels of IL-2 and IL-4 were unaffected by SULF A in T cells alone (Supplementary Fig. 11), thus indicating that their modulation was strictly related to the trigger of SULF A on DC maturation. Furthermore, according to the lack of pro-inflammatory activity by SULF A, we also found that the sulfolipid did not affect release of Th1 cytokines, namely IL-12 and IFNg, in the MLR system (Fig. 5F-G). These results support the unconventional maturation of DC and the resulting regulatory immune response induced by SULF A through TREM2 engagement.

\section{Discussion}

This study demonstrates that TREM2 mediates the effects of SULF A on DC maturation. In agreement with the regulatory function of this receptor, the stimulation by SULF A prompted the differentiation of DC towards a novel homeostasis-determining phenotype (homeDCs) displaying hypoproduction of cytokines and up-regulation of the costimulatory and class II Ag-presenting molecules. The signal induced by the sulfolipid is correlated to the TREM2 expression on the membrane of $h$-MoDCs since both antibody blocking and down-regulation of the receptor transcription inhibited DC maturation in a dosedependent manner. This loss-of-function is similar to the hyporesponsiveness to putative ligands due to either TREM2 shedding by g-secretase (also known as sheddase) [38] or to the reduced availability of TREM2 on cell surface by pathogenic mechanisms due to mutations or incorrect glycosylation.

Colonna and co-workers first pointed out that TREM2 on microglia can sense negatively charged lipids as an "eat-me" signal for professional phagocytes [17]. In line with this view, two very recent studies report that mycolic acid derivatives and aminophospholipids, such as phosphatidylserine (PS) and phosphatidylethanolamine, can trigger TREM2 signalling in apoptotic cells and macrophages $[18,19]$. 
SULF A (Figure 1) is a sulfoquinovoside glycerolipid with two saturated fatty acid chains that resemble phospholipids of bacterial membranes [16]. The sulfoquinovoside group mimics the polar head of phospholipids but the strong acidic property of the sulfonic acid increases the negative charge density in comparison to phospholipids. According to the current model of binding to TREM2 [17, 39], this is expected to promote the interaction with the receptor thus explaining the stronger signal compared to PS and other bona fide ligands (Fig. 2B). The R47H variant of TREM2 shows a rare mutation within the ligand-binding region of the receptor, which abrogates the response to PS and other putative ligands [17, 40] and increases the risk of neurodegenerative pathologies as Alzheimer diseas e[12, 41]. The reduced affinity of SULF A for the mutant receptor (Fig. 2D) is clear evidence of the capacity to structurally mimic the binding affinity of endogenous ligands. It is also worth noting that the sugar head of SULF A resembles the structure of the cyclic polyol pinitol, a natural product of pharmacological interest that is suggested to ameliorate inflammation by binding to TREM2 in macrophages and microglia [42].

TREM2 signalling via DAP12 promotes survival and maturation of human DCs [25] but the absence of specific ligands has so far hampered fine characterization of the receptor function. SULF A induced a transient up-regulation of the expression of SYK and NFATc genes within $3 \mathrm{~h}$ from the stimulation, which is in line with the previous reports on the downstream intracellular pathway of TREM2 (Fig. 2E). In line with the low proinflammatory response induced by SULF A, the NF-kB pathways that are classical effectors of TLR-based adjuvants were not affected by the sulfolipid. The NFAT genes appeared recently in the evolution in order to cope with the increasing complexity of the immune response in vertebrates, including control of inflammatory process triggered by PRRs and collaboration between innate and adaptive immunity [43]. The NFATc function is tightly regulated by $\mathrm{Ca}^{2+}$-dependent post-translational modifications but the SULF A-induced overexpression of NFAT1 suggests other forms of control that deserve further studies in consideration of the emerging role of this group of transcriptional factors in various physiological and pathological processes.

The regulatory mechanism triggered by SULF A was proven in the context of the inflammatory response to LPS (Fig. 5A). Stimulation of DC with SULF A did not reduce the LPS-dependent release of proinflammatory cytokines but boosted synthesis of IL-10 (Fig. 5B) with a slight downregulation of the surface expression of CD86, CD83 and HLA-DR (Supplementary Fig. 10). This DC phenotype is different from that generated by SULF A alone and, in our opinion, is committed to the attenuation of the inflammatory stimulus. In fact, IL-10 is a regulatory cytokine that is produced with delayed kinetics compared to proinflammatory cytokines in order to prevent chronic inflammation and tissue damage due to an excessive immune response [31, 32]. It is worth noting that initiation of the anti-inflammatory pathway by LPS is driven by NFAT1 and NFAT3 in DCs [44], while production of IL-10 requires the contribution of NFATc members after dectin-1 engagement in macrophages and DCs [45]. This regulatory function of SULF A fits well with a general characteristic of TREM2 whose signalling is critically dependent on ligands, intracellular state and tissue context [11]. The mediated effect of SULF A on T cells in an allogeneic MLR further supports the differentiation of an unconventional DC phenotype mostly committed to the maintenance of homeostasis and tuning of the immune response. T cell priming is a

Page $12 / 25$ 
hallmark of DC function and the MLR is a consolidated in vitro model for studying the mechanisms of allo-responsiveness. Thus, it is significant that SULF A increased expansion of DC-dependent T cells and synthesis of IL-4 and IL-2 (Fig. 5D-E)without affecting the levels of pro-inflammatory cytokines such as IL12 and IFNg (Fig. 5F-G).

In conclusions, we described that SULF A stimulates the unconventional maturation of a DC subtype with homeostatic or regulatory functions. This activity is mediated by the engagement of TREM2. In consideration of the role of $D C$ in the positive or negative modulation of leukocyte response, the activity of SULF A may lay the groundwork for the development of a new class of drugs with therapeutic potential in chronic-inflammation and cancer [46-49]. Furthermore, SULF A is the first synthetic small molecule targeting TREM2 selectively. This finding warrants the investigation of this product in diseases well known to be bound to TREM-2 dysfunction, such as chronic and neurodegenerative diseases[11-14].

\section{Declarations}

\section{Statements and Declarations}

\section{Funding}

This research was supported by the project "Antitumor Drugs and Vaccines from the Sea (ADViSE)" (CUP B43D18000240007-SURF 17061BP000000011; PG/2018/0494374) funded by POR Campania FESR 2014-2020 “Technology Platform for Therapeutic Strategies against Cancer"-Action 1.1.2 and 1.2.2.

\section{Competing interests}

The authors declare no competing interests.

\section{Author contributions}

CG and GB prepared and performed the experiments. EM, LF and $M Z$ carried out the preparation, formulation and analysis of sulfavant A. GN, Gdl, FF and PC supported the experimental work and analysis; CG, GB, EM collected the data. CG, EM and AF analysed the results. CA performed the statistical analysi. RdP contributed to the interpretation of the results and, together AF, conceived the study. AF planned the experiments and, together with $C G$, wrote the draft of the manuscript. All authors read, commented on and approved the final manuscript.

\section{Data Availability}

All data generated or analysed during this study are included in this published article and its supplementary information files. Further information is available from the corresponding author on reasonable request.

\section{Ethics approval}


This is an observational study and no ethical approval is required. The data utilized human tissue that was procured via Presidio Ospedaliero Umberto I of Nocera Inferiore (Salerno, Italy) and the Trasfusional Center of II Policlinico (Naples, Italy), which provides de-identified samples. The study is in accordance with the ethical standards of our institution and with the 1964 Helsinki declaration and its later amendments or comparable ethical standards.

\section{Consent to participate}

This study does not involve human subjects.

\section{Consent to Publish}

The study doe not involve human participants and no consent for publication of images is required.

\section{Acknowledgments}

This research was supported by the project "Antitumor Drugs and Vaccines from the Sea (ADViSE)" (CUP B43D18000240007-SURF 17061BP000000011; PG/2018/0494374) funded by POR Campania FESR 2014-2020 "Technology Platform for Therapeutic Strategies against Cancer"-Action 1.1.2 and 1.2.2. Authors are grateful to Dr. Carmine Oricchio and Dr. Caterina Del Gaudio of Presidio Ospedaliero Umberto I of Nocera Inferiore, Salerno, Italy and to prof. Antonio Leonardi of Trasfusional Center c/o II Policlinico, Naples, Italy for providing the human blood samples. We acknowledge BioSEArch SRL for the permission to use Sulfavant A. RdP and AF thank Prof. Marco Colonna and Prof Marina Cella of the Washington University School of Medicine in St. Louis (USA) for the graceful gift of TREM2 and R47H TREM2 reporter cell lines. The authors also thank Dr. Lucio Caso and Dr Salvatore Morra for the technical support.

\section{References}

1. Banchereau J, Steinman RM (1998) Dendritic cells and the control of immunity. Nature 392:245252. https://doi.org/10.1038/32588

2. Mellman I (2013) Dendritic cells: master regulators of the immune response. Cancer Immunol Res 1:145-149. https://doi.org/10.1158/2326-6066.CIR-13-0102

3. Kim MK, Kim J (2019) Properties of immature and mature dendritic cells: phenotype, morphology, phagocytosis, and migration. RSC Adv 9:11230-11238. https://doi.org/10.1039/C9RA00818G

4. Rossi M, Young JW (2005) Human Dendritic Cells: Potent Antigen-Presenting Cells at the Crossroads of Innate and Adaptive Immunity. J Immunol 175:1373-1381.

https://doi.org/10.4049/jimmunol.175.3.1373

5. Tisch R (2010) Immunogenic versus tolerogenic dendritic cells: A matter of maturation. Int Rev Immunol 29:111-118. https://doi.org/10.3109/08830181003602515 
6. Domogalla MP, Rostan P V., Raker VK, Steinbrink K (2017) Tolerance through education: How tolerogenic dendritic cells shape immunity. Front Immunol 8:1-14.

https://doi.org/10.3389/fimmu.2017.01764

7. Manzo E, Cutignano A, Pagano D, et al (2017) A new marine-derived sulfoglycolipid triggers dendritic cell activation and immune adjuvant response. Sci Rep 7:6286. https://doi.org/10.1038/s41598017-05969-8

8. Manzo E, Gallo C, Fioretto L, et al (2019) Diasteroselective Colloidal Self-Assembly Affects the Immunological Response of the Molecular Adjuvant Sulfavant. ACS Omega 4:7807-7814. https://doi.org/10.1021/acsomega.8b03304

9. Khameneh HJ, Ho AWS, Spreafico R, et al (2017) The Syk-NFAT-IL-2 Pathway in Dendritic Cells Is Required for Optimal Sterile Immunity Elicited by Alum Adjuvants. J Immunol 198:196-204. https://doi.org/10.4049/jimmunol.1600420

10. Mócsai A, Ruland J, Tybulewicz VLJ (2010) The SYK tyrosine kinase: A crucial player in diverse biological functions. Nat Rev Immunol 10:387-402. https://doi.org/10.1038/nri2765

11. Deczkowska A, Weiner A, Amit I (2020) The Physiology, Pathology, and Potential Therapeutic Applications of the TREM2 Signaling Pathway. Cell 181:1207-1217. https://doi.org/10.1016/j.cell.2020.05.003

12. Ulland TK, Colonna M (2018) TREM2 - a key player in microglial biology and Alzheimer disease. Nat Rev Neurol 14:667-675. https://doi.org/10.1038/s41582-018-0072-1

13. Colonna M, Wang Y (2016) TREM2 variants: new keys to decipher Alzheimer disease pathogenesis. Nat Rev Neurosci 17:201-207. https://doi.org/10.1038/nrn.2016.7

14. Humphrey MB, Xing J, Titus A (2015) The TREM2-DAP12 signaling pathway in Nasu-Hakola disease: a molecular genetics perspective. Res Reports Biochem 5:89. https://doi.org/10.2147/RRBC.S58057

15. Quan DN, Cooper MD, Potter JL, et al (2008) TREM-2 binds to lipooligosaccharides of Neisseria gonorrhoeae and is expressed on reproductive tract epithelial cells. Mucosal Immunol 1:229-238. https://doi.org/10.1038/mi.2008.1

16. Daws MR, Sullam PM, Niemi EC, et al (2003) Pattern Recognition by TREM-2: Binding of Anionic Ligands. J Immunol 171:594-599. https://doi.org/10.4049/jimmunol.171.2.594

17. Wang $Y$, Cella M, Mallinson $\mathrm{K}$, et al (2015) TREM2 lipid sensing sustains the microglial response in an Alzheimer's disease model. Cell 160:1061-1071. https://doi.org/10.1016/j.cell.2015.01.049 
18. Shirotani K, Hori Y, Yoshizaki R, et al (2019) Aminophospholipids are signal-transducing TREM2 ligands on apoptotic cells. Sci Rep 9:1-9. https://doi.org/10.1038/s41598-019-43535-6

19. lizasa E, Chuma $Y$, Uematsu $T$, et al (2021) TREM2 is a receptor for non-glycosylated mycolic acids of mycobacteria that limits anti-mycobacterial macrophage activation. Nat Commun 12:2299. https://doi.org/10.1038/s41467-021-22620-3

20. Song W, Hooli B, Mullin K, et al (2017) Alzheimer's disease-associated TREM2 variants exhibit either decreased or increased ligand-dependent activation. Alzheimer's Dement 13:381-387. https://doi.org/10.1016/j.jalz.2016.07.004

21. Yao H, Coppola K, Schweig JE, et al (2019) Distinct Signaling Pathways Regulate TREM2 Phagocytic and NFkB Antagonistic Activities. Front Cell Neurosci 13:457. https://doi.org/10.3389/fncel.2019.00457

22. Ji KY, Kim SM, Yee SM, et al (2021) Cyclophilin A is an endogenous ligand for the triggering receptor expressed on myeloid cells-2 (TREM2). FASEB J 35:1-12. https://doi.org/10.1096/fj.202002325RR

23. Brown GD, Willment JA, Whitehead L (2018) C-type lectins in immunity and homeostasis. Nat Rev Immunol 18:374-389. https://doi.org/10.1038/s41577-018-0004-8

24. Kalia N, Singh J, Kaur M (2021) The role of dectin-1 in health and disease. Immunobiology 226:152071. https://doi.org/10.1016/j.imbio.2021.152071

25. Bouchon A, Hernández-Munain C, Cella M, Colonna M (2001) A Dap12-Mediated Pathway Regulates Expression of Cc Chemokine Receptor 7 and Maturation of Human Dendritic Cells. J Exp Med 194:1111-1122. https://doi.org/10.1084/jem.194.8.1111

26. Tsang E, Giannetti AM, Shaw D, et al (2008) Molecular Mechanism of the Syk Activation Switch. J Biol Chem 283:32650-32659. https://doi.org/10.1074/jbc.M806340200

27. Chae C-S, Kim G-C, Park ES, et al (2017) NFAT1 Regulates Systemic Autoimmunity through the Modulation of a Dendritic Cell Property. J Immunol 199:3051-3062.

https://doi.org/10.4049/jimmunol.1700882

28. Barboza BA, Fonseca BPF, Viola JPB (2014) NFAT1 transcription factor in dendritic cells is required to modulate T helper cell differentiation. Immunobiology 219:704-712.

https://doi.org/10.1016/j.imbio.2014.05.001

29. Murakami M, Kamimura D, Hirano T (2019) Pleiotropy and Specificity: Insights from the Interleukin 6 Family of Cytokines. Immunity 50:812-831. https://doi.org/10.1016/j.immuni.2019.03.027 
30. Liu W, Taso O, Wang R, et al (2020) Trem2 promotes anti-inflammatory responses in microglia and is suppressed under pro-inflammatory conditions. Hum Mol Genet 29:3224-3248. https://doi.org/10.1093/hmg/ddaa209

31. Ip WKE, Hoshi N, Shouval DS, et al (2017) Anti-inflammatory effect of IL-10 mediated by metabolic reprogramming of macrophages. Science (80- ) 356:513-519. https://doi.org/10.1126/science.aal3535

32. Ma X, Yan W, Zheng H, et al (2015) Regulation of IL-10 and IL-12 production and function in macrophages and dendritic cells. F1000Research 4:1-13. https://doi.org/10.12688/f1000research.7010.1

33. Couper KN, Blount DG, Riley EM (2008) IL-10: The Master Regulator of Immunity to Infection. J Immunol 180:5771-5777. https://doi.org/10.4049/jimmunol.180.9.5771

34. Eisenbarth SC (2019) Dendritic cell subsets in T cell programming: location dictates function. Nat Rev Immunol 19:89-103. https://doi.org/10.1038/s41577-018-0088-1

35. Tacken PJ, De Vries IJM, Torensma R, Figdor CG (2007) Dendritic-cell immunotherapy: From ex vivo loading to in vivo targeting. Nat Rev Immunol 7:790-802. https://doi.org/10.1038/nri2173

36. Ross SH, Cantrell DA (2018) Signaling and Function of Interleukin-2 in T Lymphocytes. Annu Rev Immunol 36:411-433. https://doi.org/10.1146/annurev-immunol-042617-053352

37. Gadani, Sachin P; Cronk J (2013) Interleukin-4: A Cytokine to Remember. J Immunol 189:42134219. https://doi.org/10.4049/jimmunol.1202246.Interleukin-4

38. Wunderlich P, Glebov K, Kemmerling N, et al (2013) Sequential Proteolytic Processing of the Triggering Receptor Expressed on Myeloid Cells-2 (TREM2) Protein by Ectodomain Shedding and YSecretase-dependent Intramembranous Cleavage. J Biol Chem 288:33027-33036. https://doi.org/10.1074/jbc.M113.517540

39. Kober DL, Alexander-Brett JM, Karch CM, et al (2016) Neurodegenerative disease mutations in TREM2 reveal a functional surface and distinct loss-of-function mechanisms. Elife 5:1-24. https://doi.org/10.7554/eLife.20391

40. Sudom A, Talreja S, Danao J, et al (2018) Molecular basis for the loss-of-function effects of the Alzheimer's disease-associated R47H variant of the immune receptor TREM2. J Biol Chem 293:1263412646. https://doi.org/10.1074/jbc.ra118.002352

41. Guerreiro R, Wojtas A, Bras J, et al (2013) TREM2 Variants in Alzheimer's Disease. N Engl J Med 368:117-127. https://doi.org/10.1056/NEJMoa1211851

42. Kong J, Du Z, Dong L (2020) Pinitol Prevents Lipopolysaccharide (LPS)-Induced Inflammatory Responses in BV2 Microglia Mediated by TREM2. Neurotox Res 38:96-104. 
43. Zanoni I, Granucci F (2012) Regulation and dysregulation of innate immunity by NFAT signaling downstream of pattern recognition receptors (PRRs). Eur J Immunol 42:1924-1931.

https://doi.org/10.1002/eji.201242580

44. Zanoni I, Ostuni R, Capuano G, et al (2009) CD14 regulates the dendritic cell life cycle after LPS exposure through NFAT activation. Nature 460:264-268. https://doi.org/10.1038/nature08118

45. Mourão-Sá D, Robinson MJ, Zelenay S, et al (2011) CLEC-2 signaling via Syk in myeloid cells can regulate inflammatory responses. Eur J Immunol 41:3040-3053. https://doi.org/10.1002/eji.201141641

46. Švajger U, Rožman PJ (2020) Recent discoveries in dendritic cell tolerance-inducing pharmacological molecules. Int Immunopharmacol 81:106275.

https://doi.org/10.1016/j.intimp.2020.106275

47. Theofilopoulos AN, Kono DH, Baccala R (2017) The multiple pathways to autoimmunity. Nat Immunol 18:716-724. https://doi.org/10.1038/ni.3731

48. Macciò A, Madeddu C (2020) Blocking inflammation to improve immunotherapy of advanced cancer. Immunology 159:357-364. https://doi.org/10.1111/imm.13164

49. Wculek SK, Cueto FJ, Mujal AM, et al (2020) Dendritic cells in cancer immunology and immunotherapy. Nat Rev Immunol 20:7-24. https://doi.org/10.1038/s41577-019-0210-z

\section{Figures}




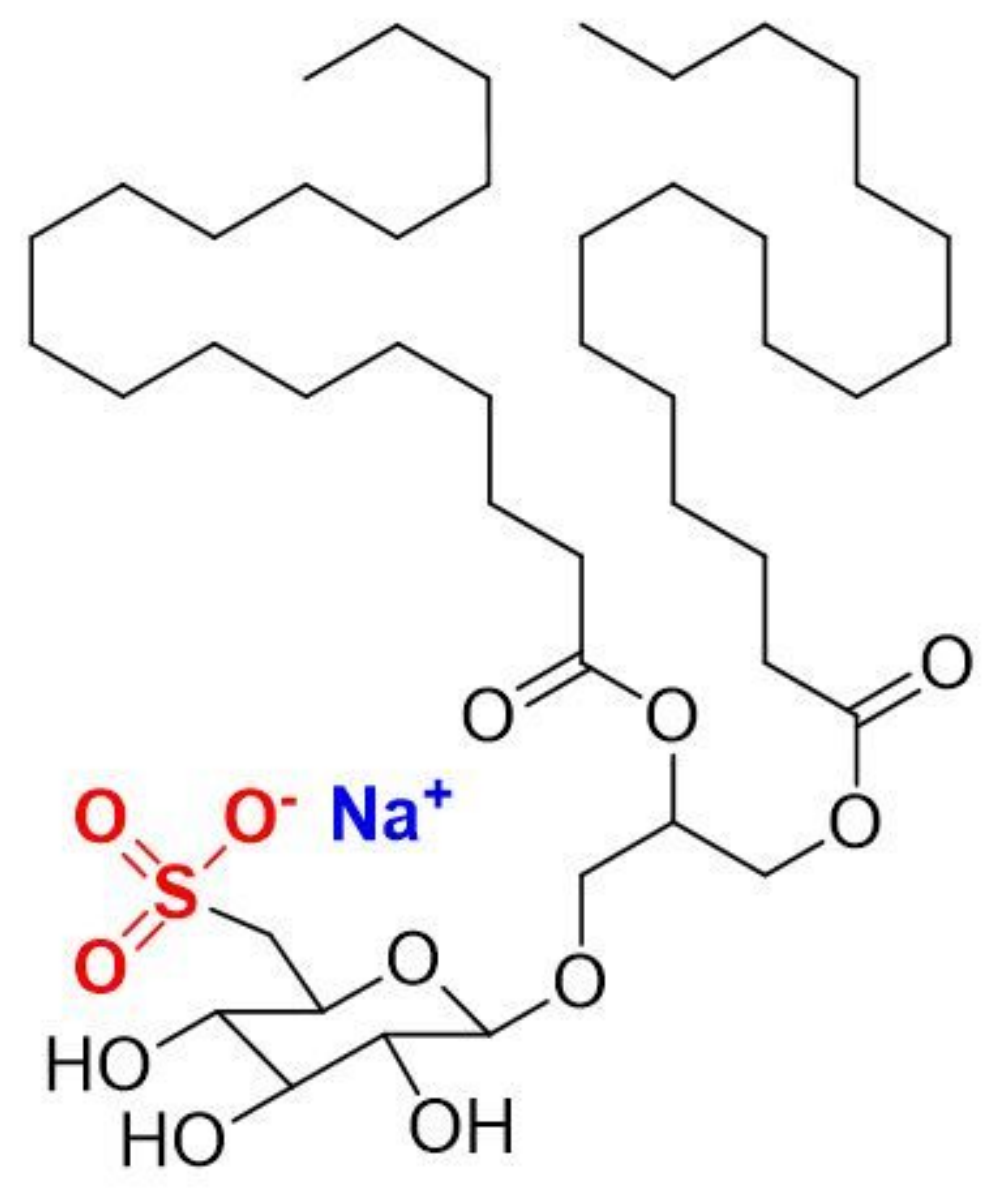

Figure 1

Structure of Sulfavant A (SULF A) with highlighting of the strong anionic sulfonate group and its counter ion. 
A)

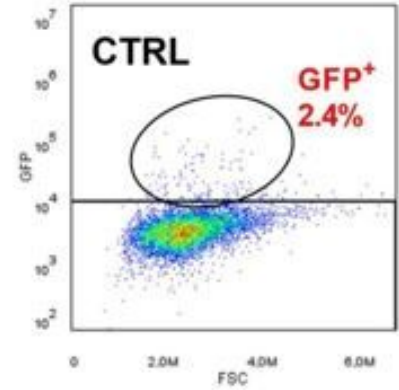

B)
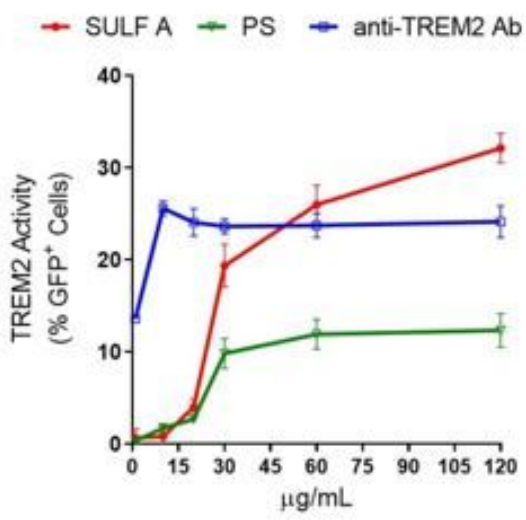

E)
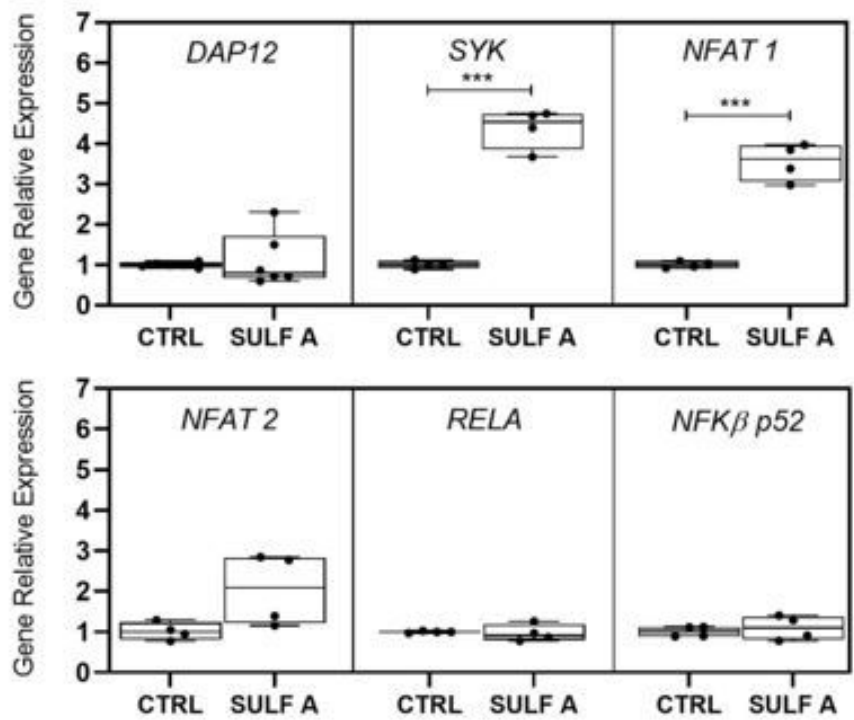
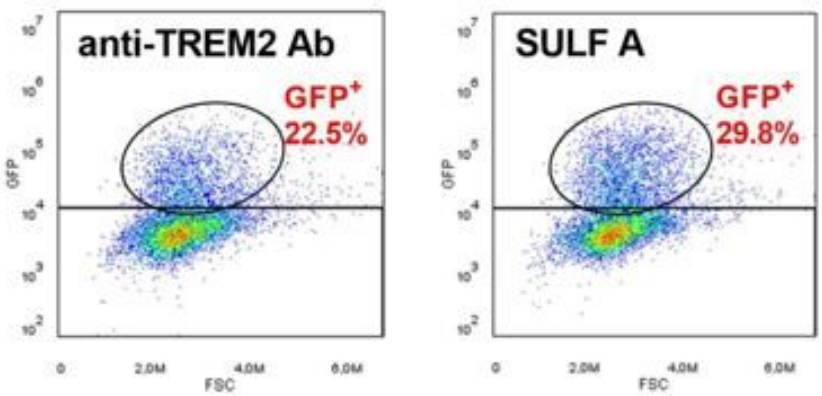

D)

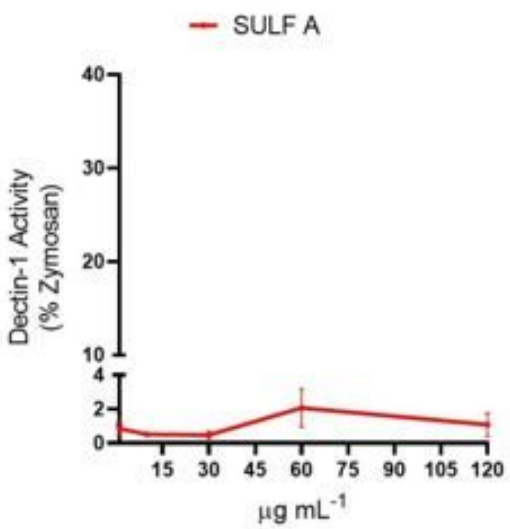

- SULFA - PS - anti-TREM2 Ab

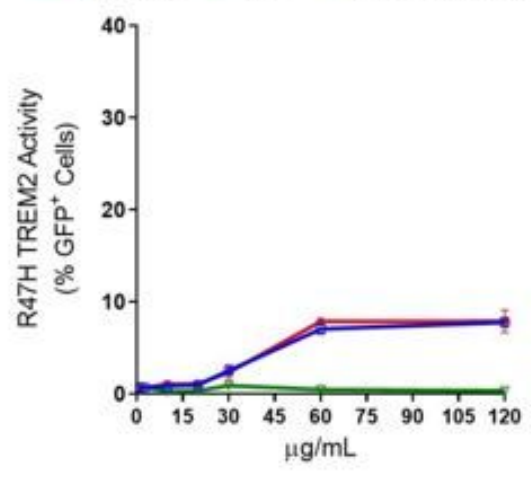

F)

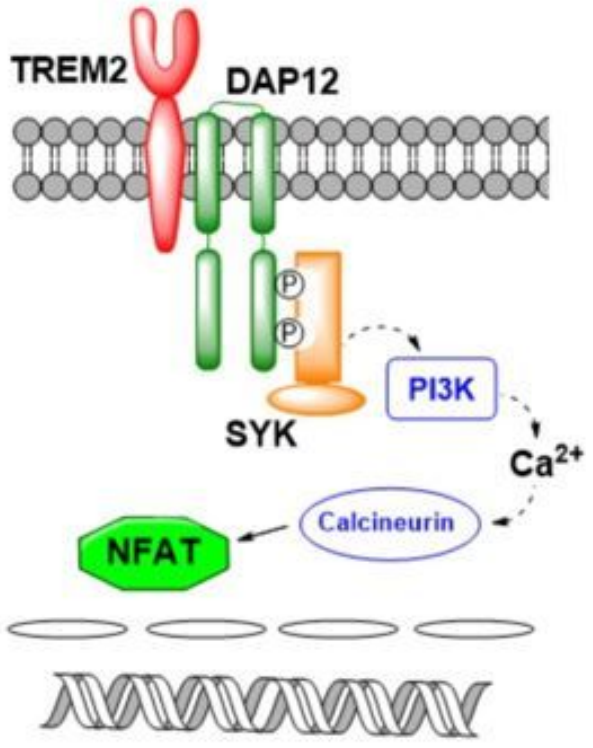

Figure 2

Engagement of SYK-coupled receptors in reporter cells and expression of intracellular TREM2 signaling molecules in $h$-MoDC by Sulfavant. CTRL = untreated reporter cells; SULF A = Sulfavant A; PS = phosphatidylserine; anti-TREM2 Ab = anti human TREM2 antibody. A) Flow cytometry analysis of GFP expression in human TREM2-reporter cell after stimulation. One representative experiment by $60 \mu \mathrm{g} / \mathrm{mL}$ PS, $10 \mu \mathrm{g} / \mathrm{mL}$ anti-TREM2 Ab, and $10 \mu \mathrm{g} / \mathrm{mL}$ SULF A is shown; B) Dose-dependent response of human 
TREM2-reporter cells $(n=4)$. Statistical analysis (see Supplementary Material) was performed using twoway RM ANOVA followed by BH correction in the post-hoc analysis; C) Dose-dependent response of Dectin 1-reporter cells $(n=3)$. Statistical analysis was performed using one-way RM ANOVA; $D)$ Dosedependent response of mutant R47H human TREM2-reporter cells $(n=3)$. Statistical analysis was carried out as in Figure 1B; E) Relative expression of the main genes related to the intracellular TREM2 pathway after $3 \mathrm{~h}$ in $h$-MoDC from four donors $(\mathrm{n}=4)$. Statistical differences were determined by paired samples $\mathrm{T}$ test (one-side alternative). Significance refers to $* \star * P<0.001 ; F)$ Proposed TREM2 signaling pathway with the main steps for the intracellular propagation of the signal via DAP12/SYK upon receptor activation
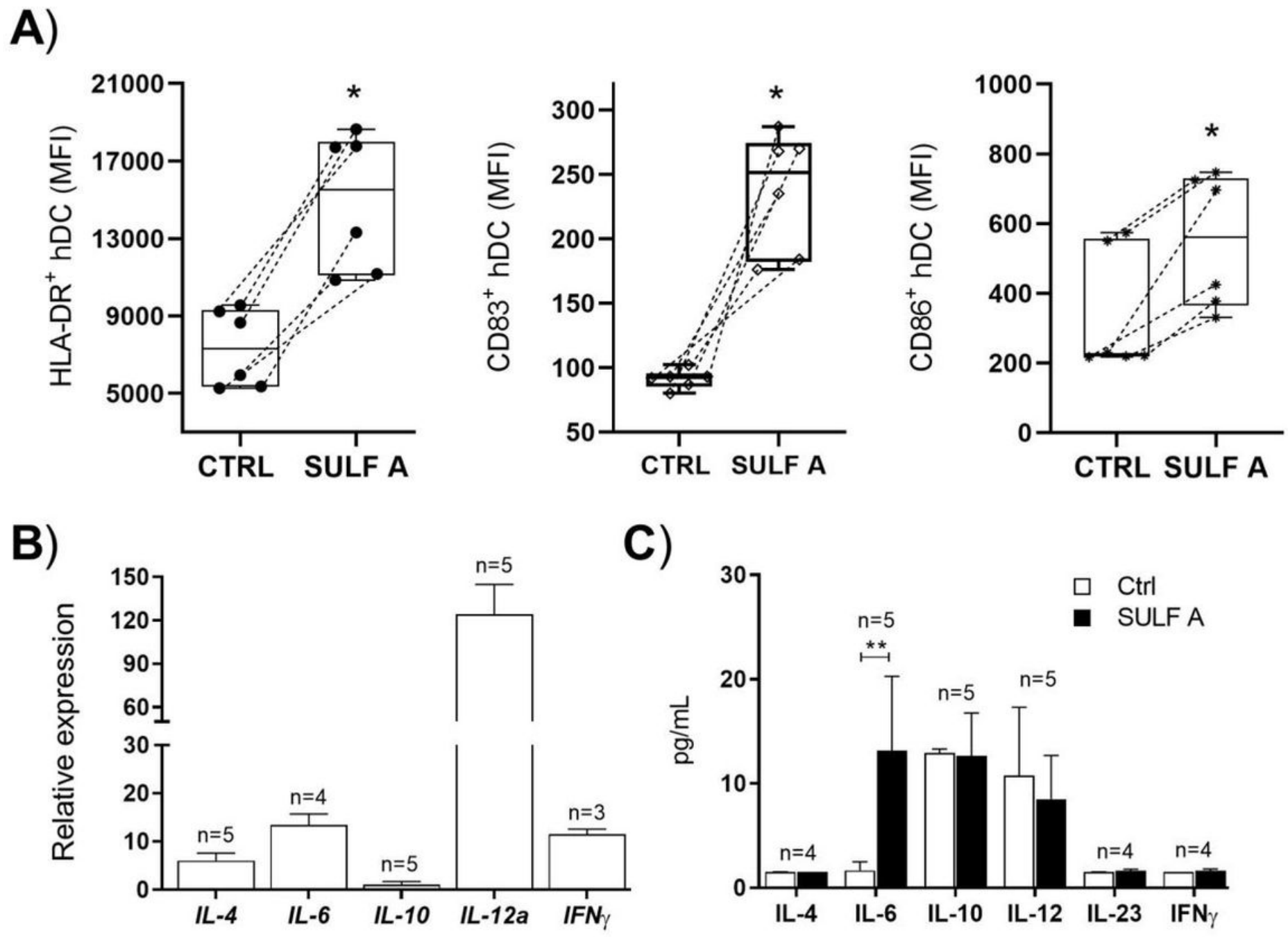

Figure 3

Maturation of $h$-MoDCs following exposure to Sulfavant A. CTRL $=$ untreated cells; SULF A $=10 \mu \mathrm{g} / \mathrm{mL}$ Sulfavant A. A) Surface expression as Mean Fluorescence Intensity (MFI) of HLA-DR, CD83 and CD86 on six matched donors $(n=6)$ at $24 \mathrm{~h}$ by flow cytometry; B) RT PCR analysis of cytokine gene expression at 
$24 \mathrm{~h} ; \mathrm{n}=$ donor replicates; C) ELISA analysis of cytokines in the supernatants at $48 \mathrm{~h} ; \mathrm{n}=$ donor replicates. Statistical significance (* $P<0.05$; $* * ~ P<0.01$ ) was established by non-parametric two-sample Wilcoxon Test (two-side alternative). Paired tests and one-side alternatives were used for marker surface expression

A)

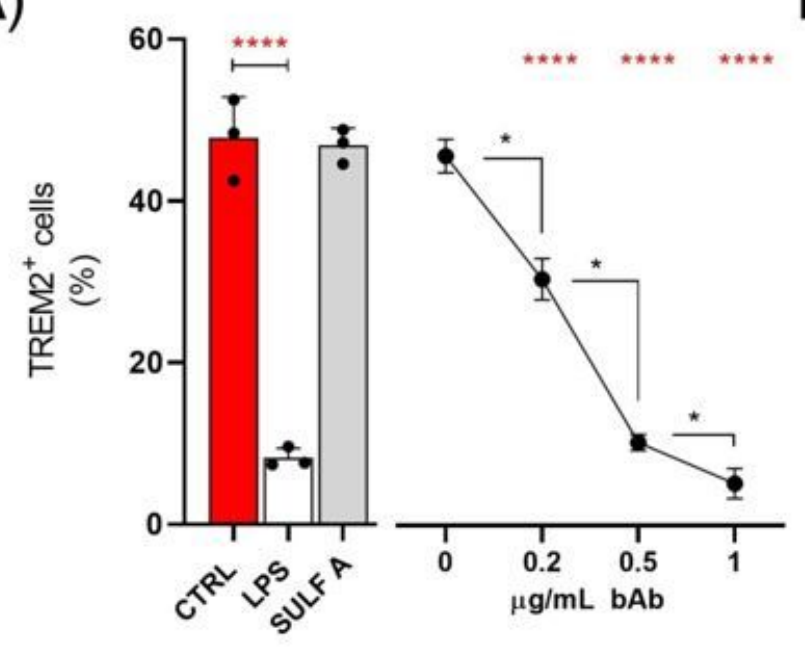

B)

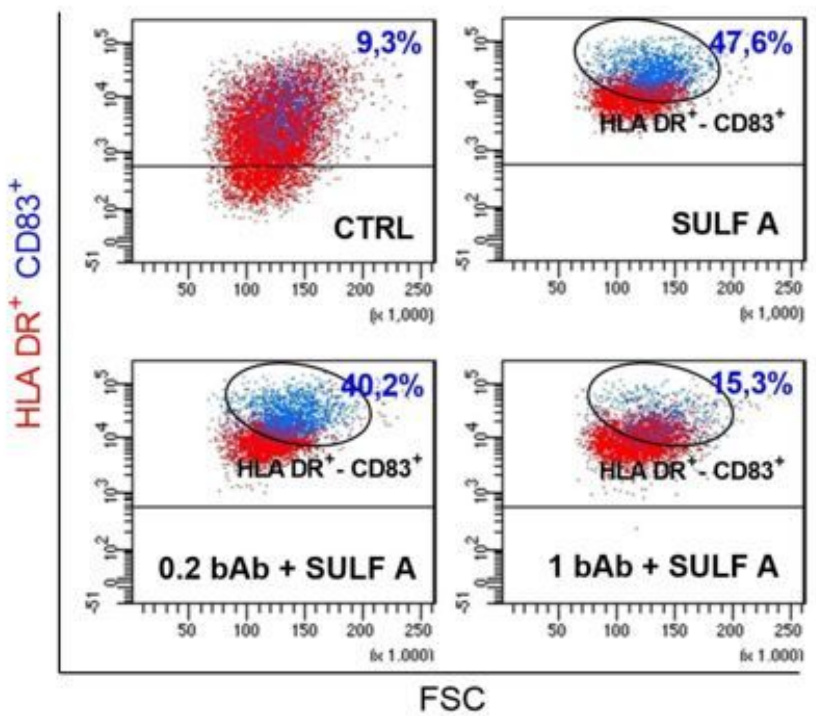

C)

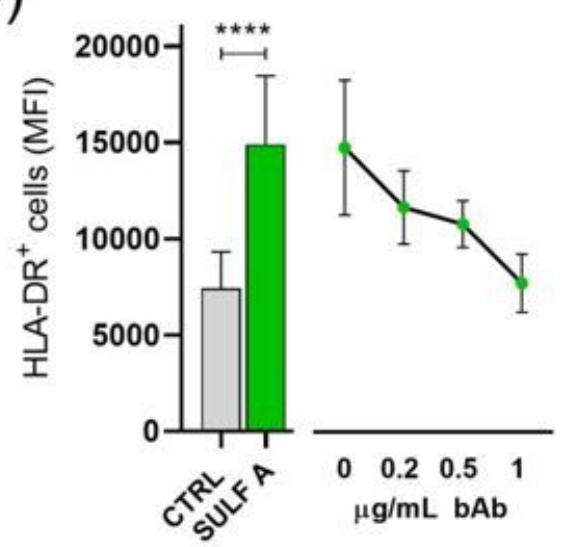

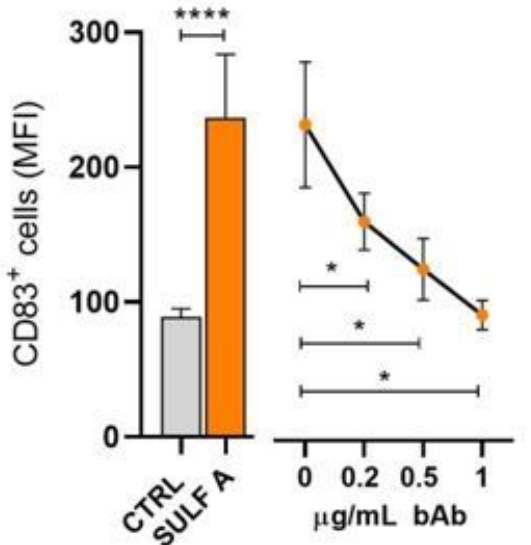

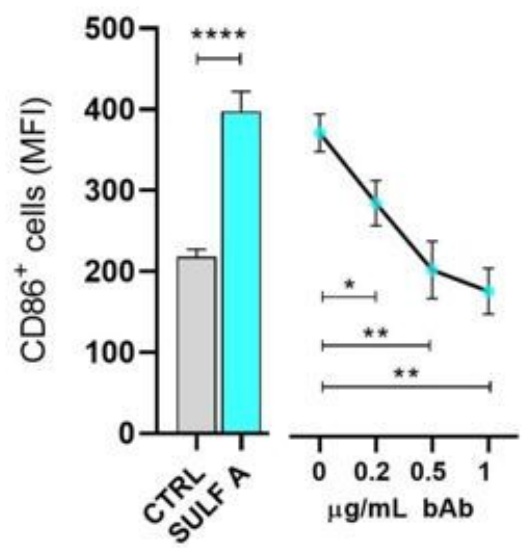

D)

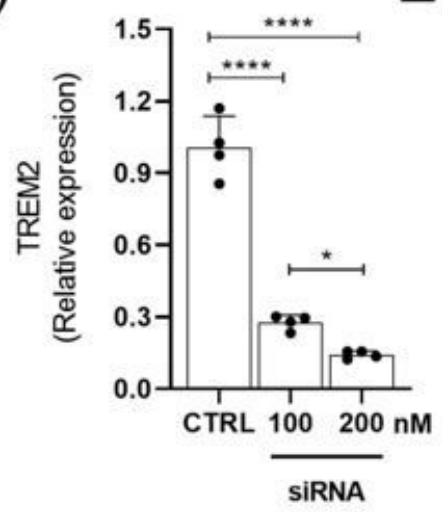

E)

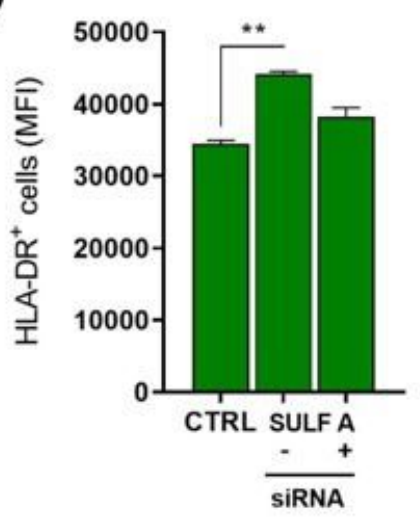

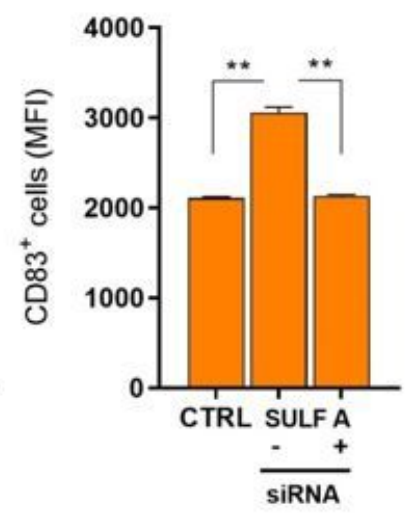

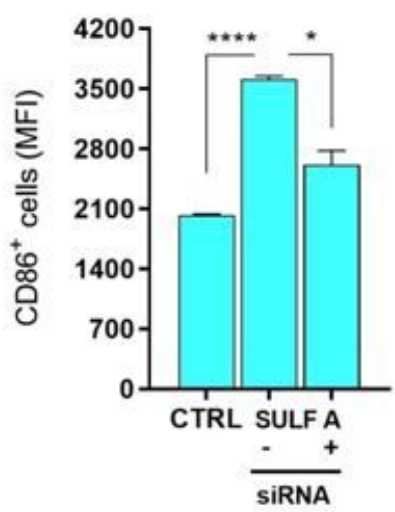

Figure 4 
Functional impairment of Sulfavant A activity by antibody blocking assay and gene silencing of TREM2

in $h$-MoDC. CTRL = untreated cells; SULF A $=10 \mu \mathrm{g} / \mathrm{mL}$ Sulfavant A; bAb = anti-TREM2- blocking antibody AF1828. A) Dose-dependent shedding of TREM2 on h-MoDCs from three donors $(n=3)$ by increasing concentration of the blocking antibody. Effect was compared to $10 \mathrm{ng} / \mathrm{mL}$ LPS; B) Flow cytometry gating analysis of HLA-DR and CD83 expression on $h$-MoDCs treated by sufavant A alone and together with $0.2 \mu \mathrm{g} / \mathrm{mL}$ and $1 \mu \mathrm{g} / \mathrm{mL}$ of anti-TREM2 blocking antibody; C) Dose-dependent reduction of HLA-DR (right), CD83 (middle) and CD86 (right) on h-MoDCs treated by Sulfavant A and increasing concentration of anti-TREM2 blocking antibody from three donors $(n=3)$; $D)$ Reduction of TREM2 gene expression by 100 and $200 \mathrm{nM}$ siRNA in $h$-MoDCs from two donors with two technical replicates $(\mathrm{n}=4)$; E) Effect of TREM2 gene silencing on the surface expression of HLA-DR (left), CD83 (middle) and CD86 (right) in h-MoDC by flow cytometry. Cells were silenced by $200 \mathrm{nM}$ siRNA prior to addition of Sulfavant A from four donors $(n=4)$. CTRL= cells treated with $200 \mathrm{nM}$ negative siRNA. Surface expression is reported as Mean Fluorescence Intensity (MFI). Statistical significance $\left({ }^{*} P<0.05 ; * \star P<0.01\right.$; $* \star * P<0.001$; $* \star \star * P$ $<0.0001$ ) was determined by one-way RM ANOVA (panel A, C and E) or LMM (panel D) followed by appropriate post-hoc analysis including Dunnett, BH or Tukey's test (see Supplementary Material) 
A)

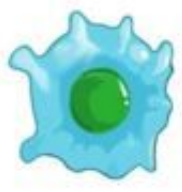

immature

h-MoDC
SULF A LPS

1 .

$0 \quad 30 \mathrm{~min}$

treatment
$24 \mathrm{~h}$

B)

IL-10

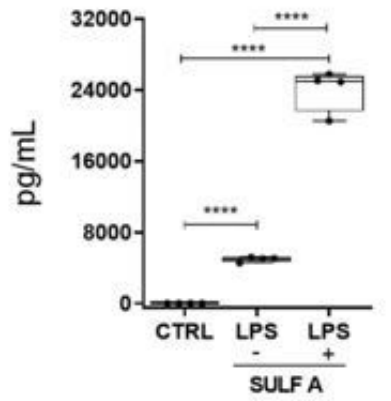

IL-12

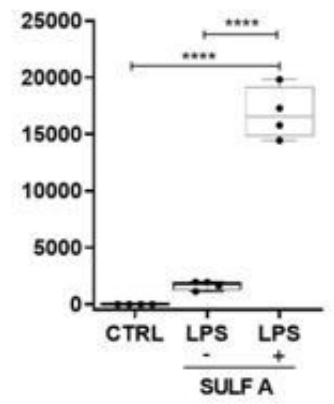

C)

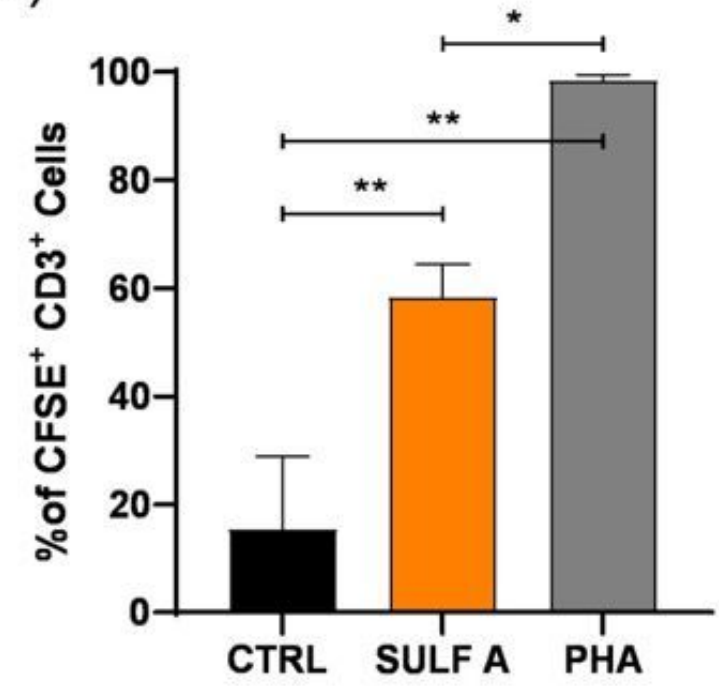

IL-23

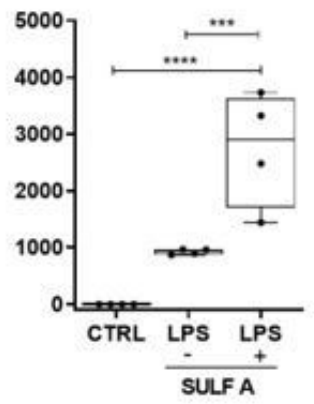

IFN $\gamma$

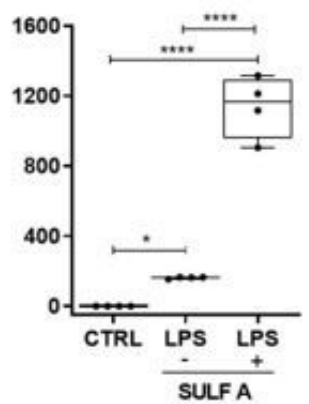

IL-6

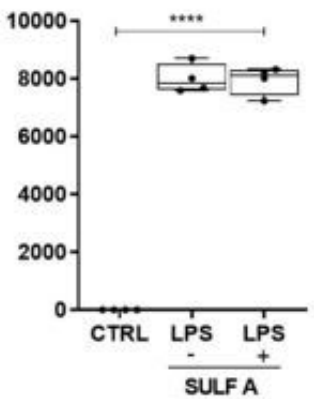

D)

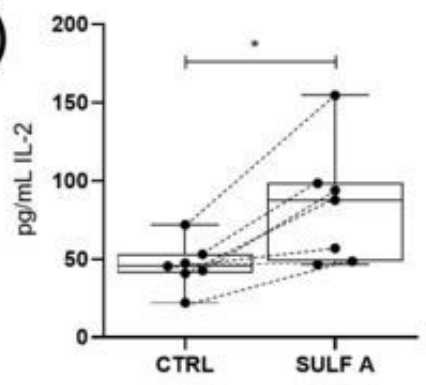

F)

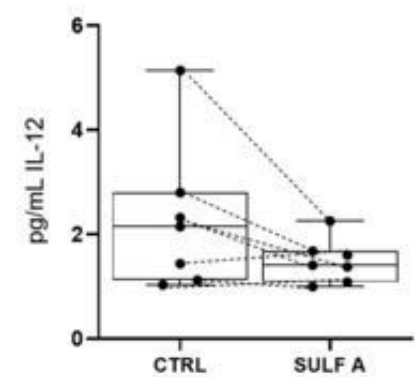

E)

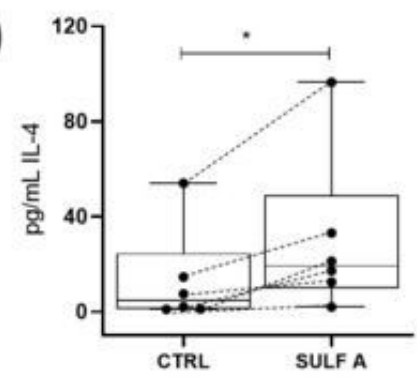

G)

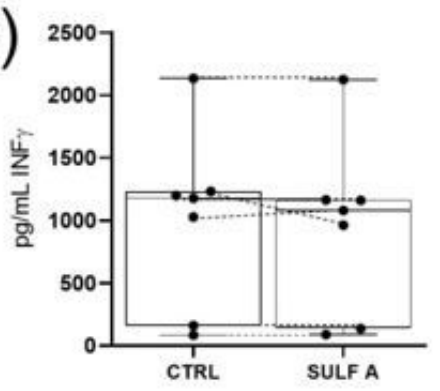

Figure 5

Sulfavant A affects TLR-dependent cytokine release in $h$-MoDCs and allogeneic MLR. CTRL $=$ untreated cells; SULF A $=10 \mu \mathrm{g} / \mathrm{mL}$ Sulfavant A. A) Design of the co-stimulation experiment by addition of 10 $\mu \mathrm{g} / \mathrm{mL}$ SULF A followed by $5 \mathrm{ng} / \mathrm{mL}$ LPS; B) ELISA analysis of cytokines in the supernatants of the cocultures after $48 \mathrm{~h}$ from two donors with two technical replicates. Statistical analysis was performed by LMM. Post-hoc analysis was carried out using Tukey test; C) Flow cytometry analysis of T lymphocytes 
expansion. CFSE-labelled T cells were co-cultured with stimulated $h$-MoDC (1:10 ratio) for 7 days. CTRL $=$ untreated co-colture; SULF A = co-colture stimulated by $10 \mu \mathrm{g} / \mathrm{mL}$ Sulfavant A; PHA = co-colture stimulated by $1 \mu \mathrm{g} / \mathrm{mL}$ phytohemagglutinin (positive control). Proliferation rate is expressed as median values of $\mathrm{CFSE}^{+} \mathrm{CD}^{+}$cells in allogeneic co-cultures from three different donors $(n=3)$. D-G) ELISA of IL-2, IL-4, IL-12 and IFNg in the supernatants of the allogeneic co-cultures. Statistical significance $(* P<0.05$; ** $\mathrm{P}<0.01$;) was performed in matched samples of different donors ( $\mathrm{n}=7$ for IL-2, IL-12 and IFNg, $n=6$ for IL-4) by paired samples Wilcoxon Test (two-side alternative). Significance refers to * $P<0.05, * * P<0.01$, $\star \star \star \mathrm{P}<0.001, * \star \star * \mathrm{p}<0.0001$

\section{Supplementary Files}

This is a list of supplementary files associated with this preprint. Click to download.

- SupportingDataStatistics.pdf 